University of Nebraska - Lincoln

DigitalCommons@University of Nebraska - Lincoln

8-20-1999

\title{
THE EFFECT OF ABUNDANCE VARIATIONS ON ESTIMATES OF THE DENSITIES OF BROAD-LINE REGION CLOUDS IN QUASARS
}

\author{
Stephanie A. Snedden \\ University of Nebraska-Lincoln, snedden@apo.nmsu.edu \\ C. Martin Gaskell \\ University of Nebraska-Lincoln, mgaskell@ucsc.edu
}

Follow this and additional works at: https://digitalcommons.unl.edu/physicsgaskell

Part of the Physics Commons

Snedden, Stephanie A. and Gaskell, C. Martin, "THE EFFECT OF ABUNDANCE VARIATIONS ON ESTIMATES OF THE DENSITIES OF BROAD-LINE REGION CLOUDS IN QUASARS" (1999). Martin Gaskell Publications. 4.

https://digitalcommons.unl.edu/physicsgaskell/4

This Article is brought to you for free and open access by the Research Papers in Physics and Astronomy at DigitalCommons@University of Nebraska - Lincoln. It has been accepted for inclusion in Martin Gaskell Publications by an authorized administrator of DigitalCommons@University of Nebraska - Lincoln. 


\title{
THE EFFECT OF ABUNDANCE VARIATIONS ON ESTIMATES OF THE DENSITIES OF BROAD-LINE REGION CLOUDS IN QUASARS
}

\author{
Stephanie A. Snedden ${ }^{1}$ and C. Martin Gaskell \\ Department of Physics and Astronomy, University of Nebraska, Lincoln, NE 68588-0111 \\ Received 1999 January 4; accepted 1999 June 21; published 1999 July 28
}

\begin{abstract}
There has been a long-standing discrepancy between the densities deduced from studies of the variability of quasar emission lines and those inferred from standard photoionization analyses. We have found that the higher metal abundances now believed to be present in most quasars lead to the higher densities being predicted by photoionization models. We explain why this is so.
\end{abstract}

Subject heading: quasars: general

\section{INTRODUCTION}

It is well known that the strength of the line C III] $\lambda 1909$ in quasars provides an upper limit to the density of the gas in which it is formed in quasars (e.g., Osterbrock 1970). For single-zone models, this sets the upper density limit of the broad-line region (BLR). For many years, the density $\left(n_{\mathrm{H}}\right)$ of BLR clouds was assumed to be $\sim 10^{9.5} \mathrm{~cm}^{-3}$, close to the critical density of $\mathrm{C}$ III] $\lambda 1909$. If the BLR density is known and the line luminosity is also known, the volume of emitting gas can be calculated. Assuming a filling factor $f$ of unity, this gives a minimum BLR size. Emission-line equivalent widths and the weakness of absorption by resonance lines suggest that $f \sim 10^{-3}$. The results of these calculations (e.g., Osterbrock 1985) implied BLR radii on the order of light months for Seyfert galaxies to many light years for higher luminosity quasars. However, in Akn 120, emission-line variability implied that the BLR size was considerably smaller than the photoionization prediction (Peterson et al. 1985). This meant that $n_{\mathrm{H}}$ must be higher than the then "canonical" $10^{9.5} \mathrm{~cm}^{-3}$. Gaskell \& Sparke (1986) used the cross-correlation of the emission line and the continuum light curves to derive BLR sizes for active galactic nuclei (AGNs) and argued that $n_{\mathrm{H}} \sim 10^{12} \mathrm{~cm}^{-3}$. Initially, there were doubts that undersampling and irregular sampling of the line and continuum variability were producing spuriously small BLR sizes, but more intense monitoring campaigns (especially those of the International AGN Watch) have confirmed the earlier results (see Gaskell 1994; White \& Peterson 1994; Peterson et al. 1998; and references therein for discussion of this). The discrepancy between densities found from emission-line variability studies and those predicted by photoionization modeling considerations therefore remains.

In early models of BLR emission, metal abundances $(Z)$ were assumed to be close to solar. Hamann \& Ferland (1993) found that much higher abundances are needed to explain the strengths of UV metal emission lines (especially $\mathrm{N} \mathrm{v}$ ). In one well-studied quasar (Q0207-398), the metal abundances $(Z)$ seem to be enhanced to at least $5 Z_{\odot}$ (Ferland et al. 1996). Studies of broad absorption lines in quasars (Korista et al. 1996) also suggest high abundances of up to $10 Z_{\odot}$. Issues such as source coverage and line saturation complicate the interpretation of broad absorption lines, but abundance enhancements such as these arise naturally in models of the chemical enrichment of the nuclei of galaxies (see Hamann \& Ferland 1993),

\footnotetext{
${ }^{1}$ Present address: Apache Point Observatory, 2001 Apache Point Road, P.O. Box 59, Sunspot, NM 88349-0059.
}

and of course stars in our Galaxy with abundances of several times solar have long been the subject of extensive studies.

As part of an ongoing study of Hubble Space Telescope (HST) Faint Object Spectrograph (FOS) and optical spectra of low-redshift quasars, we have explored the effects of higher abundances on derived physical conditions. We find that the higher abundances result in higher predicted densities and can potentially resolve the density discrepancy between line variability studies and photoionization modeling.

\section{MODEL RESULTS}

We have analyzed emission-line profiles of HST FOS spectra and ground-based optical spectra of nearby quasars. This permits a comparison of high-ionization UV lines with optical lines in the same objects. We have been using grids of photoionization models generated by CLOUDY (Ferland 1996; Ferland et al. 1998) to deduce physical conditions in the broadline region as a function of velocity. The main UV lines are Ly $\alpha \lambda 1216, C$ IV $\lambda 1549$, and C III] $\lambda 1909$. From these three lines, the density $\left(n_{\mathrm{H}}\right)$ and ionization parameter $(U)$ can be deduced (see, e.g., Mushotzky \& Ferland 1984). We ran optically thick models using the standard AGN-ionizing continuum of Ferland (1996), which is similar to that of Mathews \& Ferland (1987), for 1 and $5 Z_{\odot}$. Abundances were varied with the CLOUDY "metals" command. The results discussed here are insensitive to the precise optical depth because the highionization lines are all produced in the optically thin part of the clouds. We show a sample grid in Figure 1.

For each quasar studied, the line ratios were measured as a function of velocity. Then $n_{\mathrm{H}}$ and $U$ were found from the grids. In Figure 2, we show typical results for one of our objects (NGC 3783). Full results will be reported elsewhere (Snedden $\&$ Gaskell 1999b). We find that the deduced $U$ is insensitive to $Z$, but it can be seen that the deduced $n_{\mathrm{H}}$ increases significantly for the higher $Z$.

\section{INTERPRETATION}

The densities deduced from the grids of models are higher for $5 Z_{\odot}$ abundances because the given conditions $\left(n_{\mathrm{H}}\right.$ and $U$ ) produce a higher $\mathrm{C}$ III]/C IV ratio for higher $Z$. To investigate the cause of this, we examined a series of models of varying $Z$ to see how the ionization structure and emissivity varied. Since we are only concerned here with line ratios, we show the dependencies on depth into the cloud in terms of the hydrogen Strömgren length, which we define as the point where 


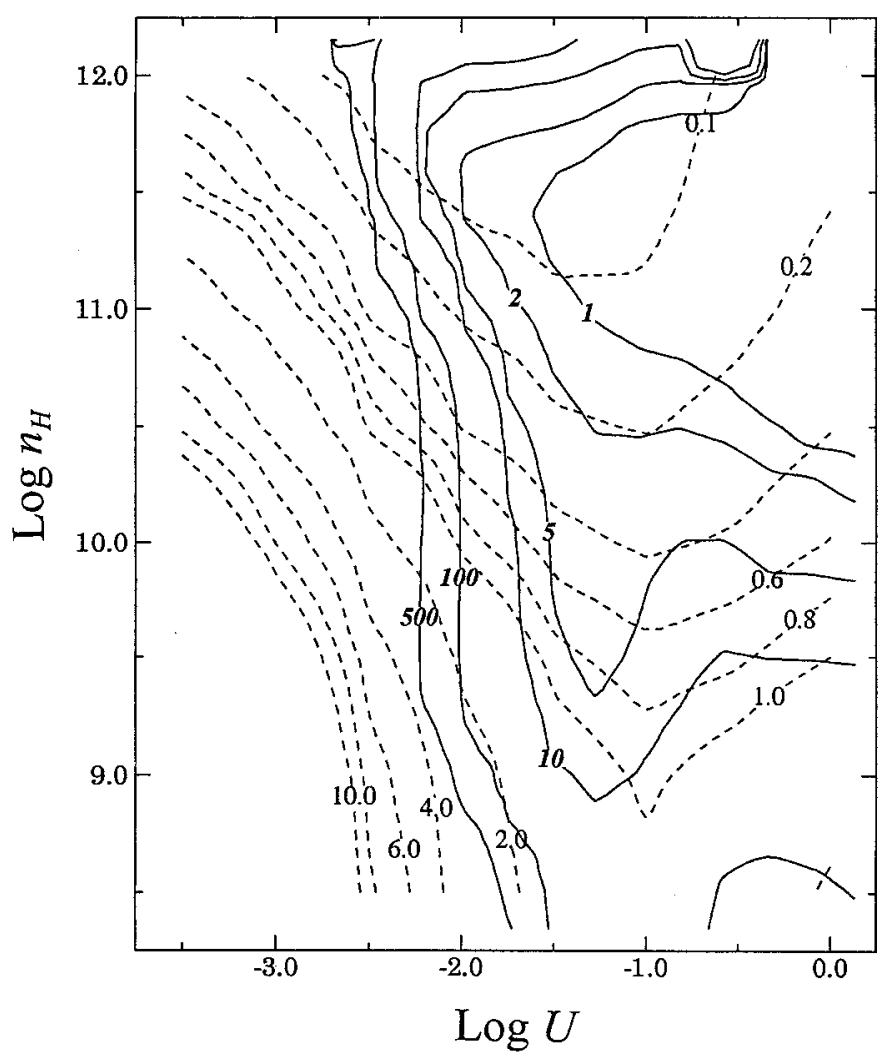

FIG. 1.-Contours of $\mathrm{C}$ III]/C IV (dashed line) and Ly $\alpha / \mathrm{C}$ IV (solid line) from CLOUDY $90.04 a$ as a function of $U$ and $n_{\mathrm{H}}$ for $5 Z_{\odot}$ optically thick clouds. The ionizing continuum is the standard AGN continuum of Ferland (1996). The line fluxes that are the sum of the emission from the front and back surfaces of the cloud were used to determine the ratios.

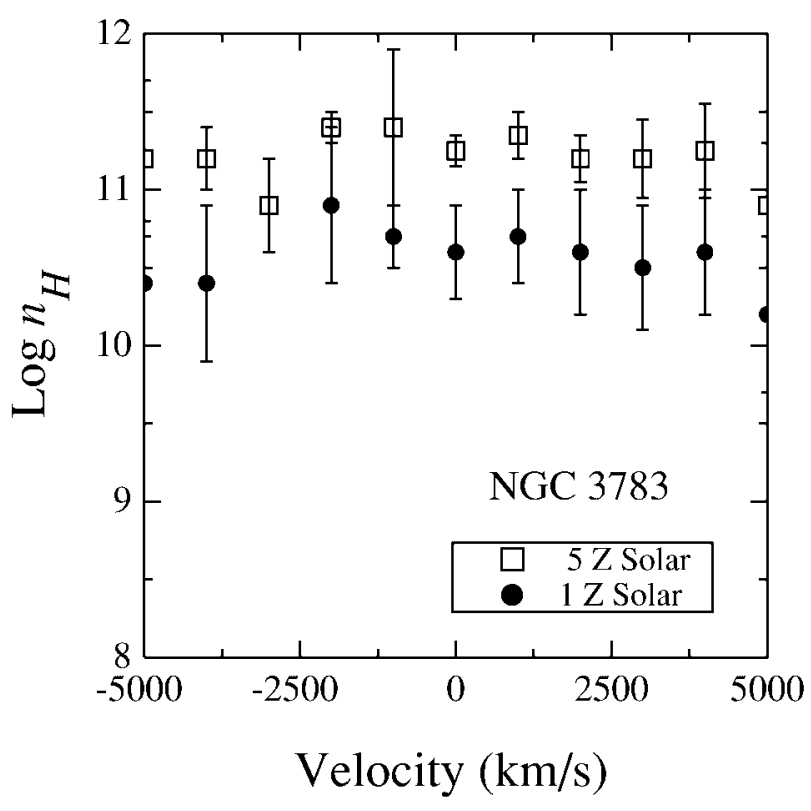

FIG. 2.-Derived $n_{\mathrm{H}}$ from $H S T$ spectra of NGC 3783. The C III]/C IV/Ly $\alpha$ emission-line ratios as a function of velocity were compared with 1 and $5 Z_{\odot}$ abundance CLOUDY models. Note that the derived $n_{\mathrm{H}}$ for the $5 Z_{\odot}$ model is almost an order of magnitude greater.

$N\left(\mathrm{H}^{+}\right) / N(\mathrm{H})$ falls to $95 \%$. Cumulative emissivities of $\mathrm{Ly} \alpha$, $\mathrm{C}$ IV, and $\mathrm{C}$ III] are shown in Figure 3, and the ionization structure of carbon, helium, and hydrogen is shown in Figure $4 .^{2}$ We find that the temperature structure at a given $U$ and $n_{\mathrm{H}}$ is essentially unchanged with varying $Z$. The main factor influencing the emissivities is the relative size of the ionization

\footnotetext{
${ }^{2}$ Note that for conditions that reproduce observed BLR line ratios, the ionization structure is different from that shown in Fig. 3 of Kwan \& Krolik (1981). This is because Kwan \& Krolik used a continuum with substantially less soft X-rays than are present in the Ferland (1996) continuum. We are able to reproduce the Kwan \& Krolik ionization and emissivity depth dependence with CLOUDY when we use their continuum.
}

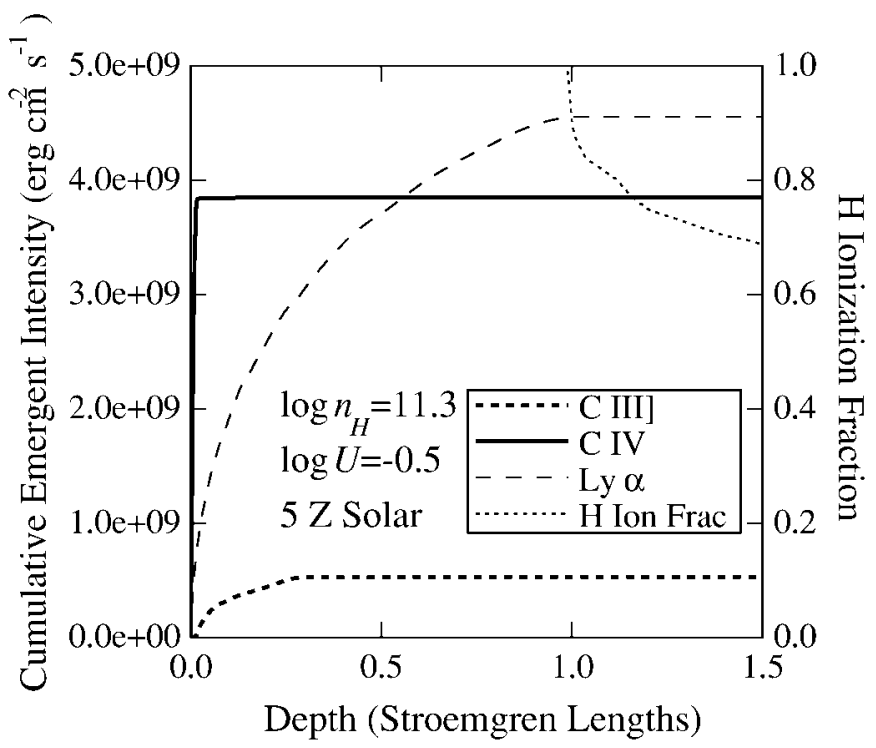

FIG. 3.-Left: CLOUDY model predictions of cumulative emergent intensities of C III], C IV, and Ly $\alpha$ for an optically thick cloud with solar abundances. Right: Emergent intensities for $5 Z_{\odot}$ clouds with the same $n_{\mathrm{H}}$ and $U$. Note that varying the abundances has a significant effect on the predicted emission-line ratios. 

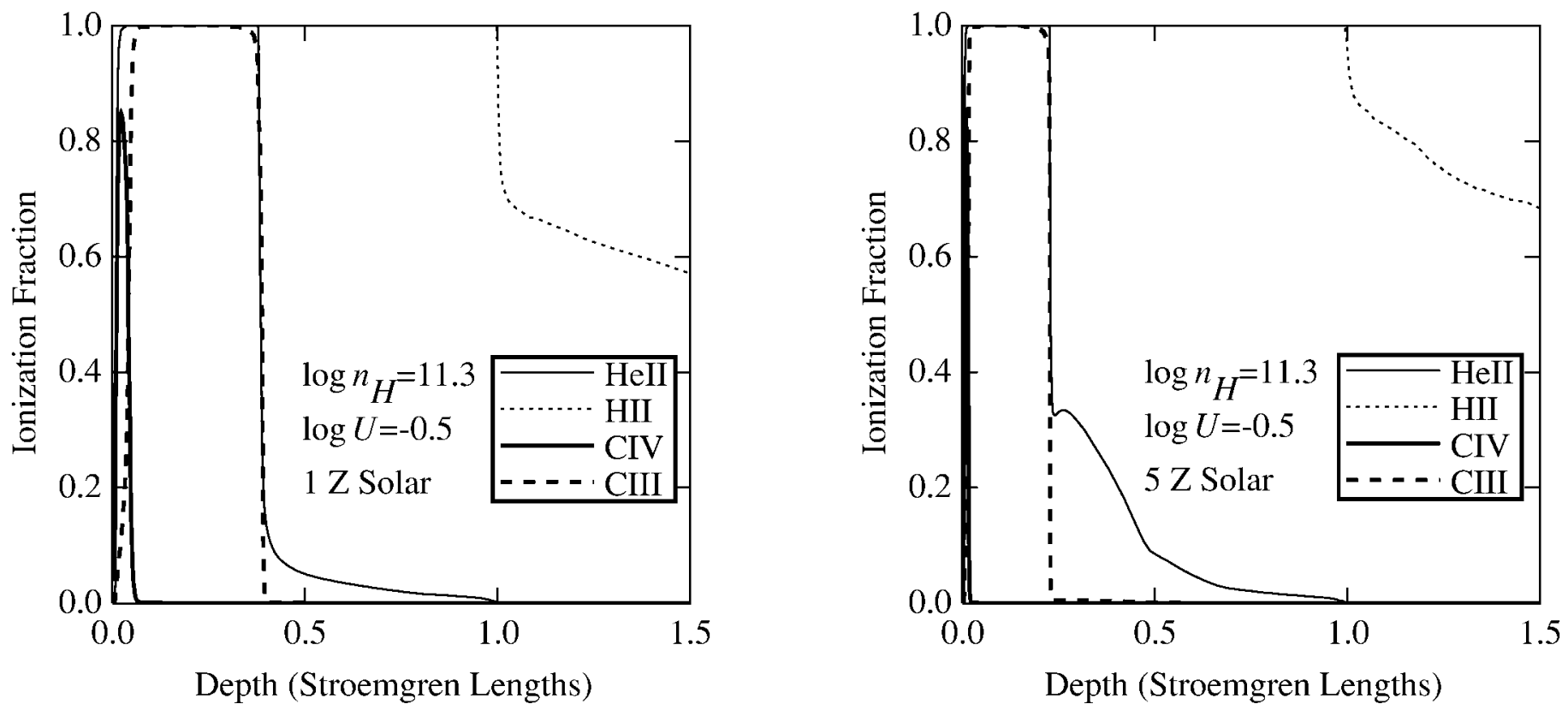

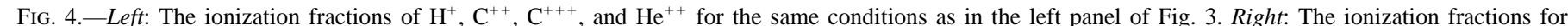
$5 Z_{\odot}$ abundances with the same conditions. Note the coupling of the $\mathrm{C}^{++}$and $\mathrm{He}^{++}$zones. The $\mathrm{C}^{+++}$zones at the extreme left are enlarged in Fig. 5 .

zones, and, as can be seen in Figure 4, these are affected by increasing $Z$.

It can be seen from Figure 4 that the position of the $\mathrm{C}^{++} /$ $\mathrm{C}^{+}$boundary is quite sharp and coincident with the $\mathrm{He}^{+} / \mathrm{He}^{0}$ boundary, independent of the carbon abundance. This is similar to the effect shown for $\mathrm{O}$ and He in Figure 2.6 of Osterbrock (1989), where the ionization edges coincide because of their similar ionization potentials. On the other hand, it can be seen from Figure 5 that the size of the $\mathrm{C}^{+++}$zone producing $\mathrm{C}$ IV varies more with the abundance. This is because the highest energy photons, which govern the ionization structure of the front of the cloud, are absorbed by the metals rather than by

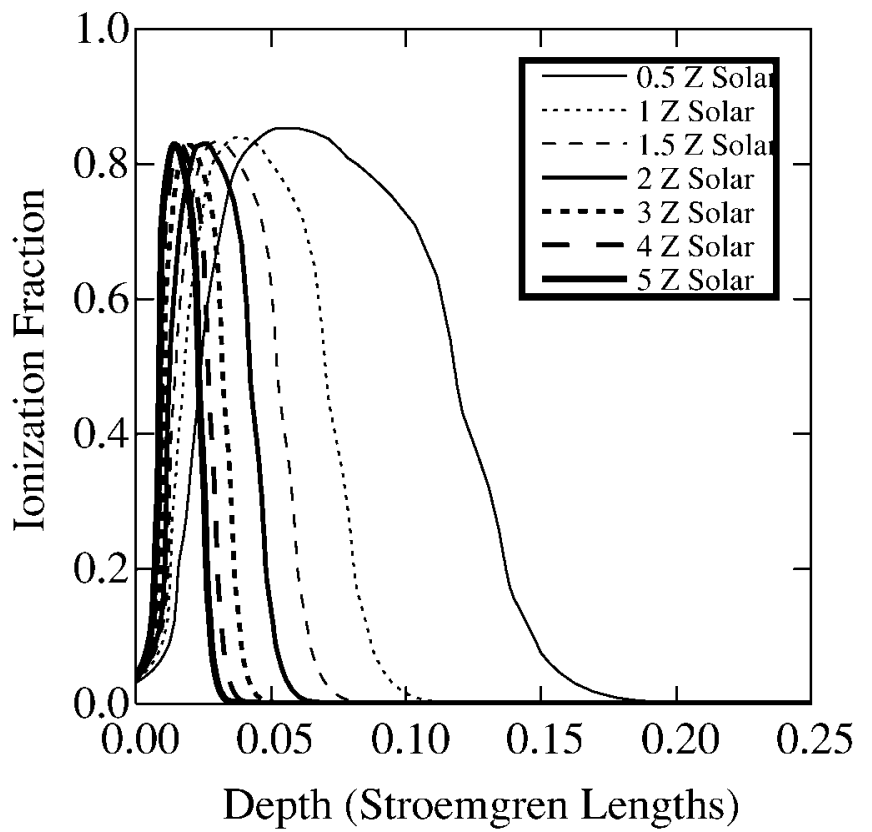

hydrogen or helium. Varying the $\mathrm{C}$ abundance alone varies the relative size of the $\mathrm{C}^{+++}$zone, and the effect is enhanced by varying all the metals.

The net result of this is that for a "hard" ionizing continuum, the relative size of the $\mathrm{C}$ III]-emitting region is insensitive to $Z$ while the relative size of the $\mathrm{C}$ IV-emitting region goes down. Thus, higher $Z$ produces a higher $\mathrm{C}$ III]/C IV ratio for the same $U$ and $n_{\mathrm{H}}$. Since the $\mathrm{C}$ III] strength decreases with density above the critical density, the higher theoretical C III]/C IV ratio for higher $Z$ implies a higher $n_{\mathrm{H}}$ for a given observed C III]/C IV ratio.

Because of their very similar ionization potentials, $\mathrm{N}^{++}$and

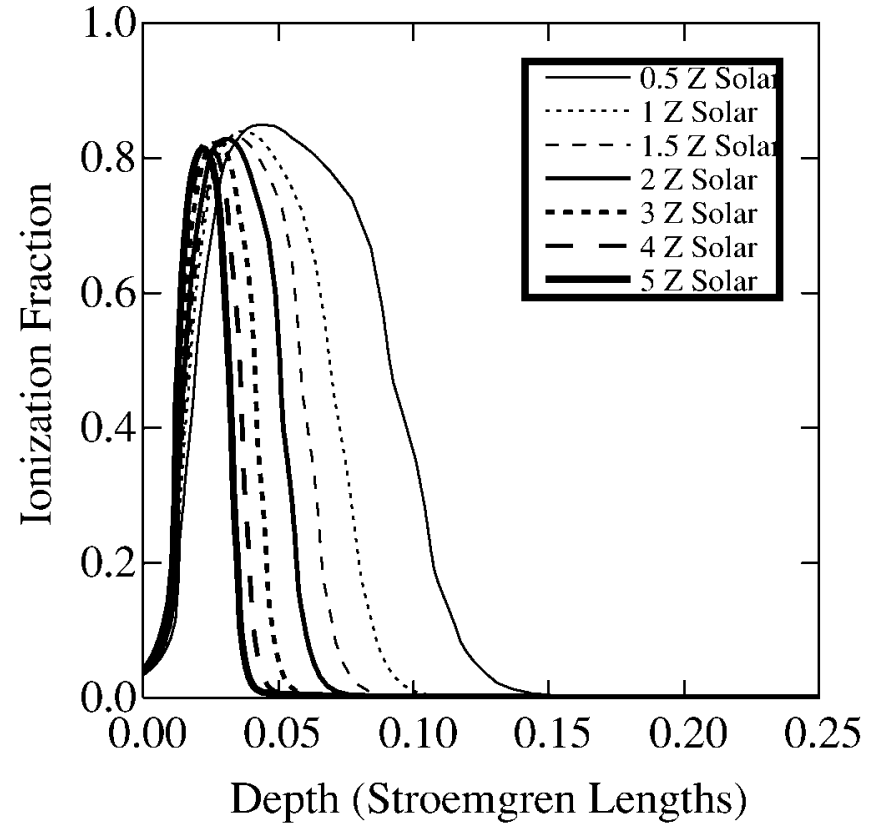


$\mathrm{N}^{+++}$have the same ionization structure as $\mathrm{C}^{++}$and $\mathrm{C}^{+++}$and show the same ionization structure dependences. Therefore, the $\mathrm{N}$ III]/ $\mathrm{N}$ IV] ratio is enhanced in the models in the same way as $\mathrm{C}$ III $] / \mathrm{C}$ IV at higher abundances. For the conditions that match $\mathrm{C} \mathrm{III]/C} \mathrm{IV/Ly} \alpha$ for NGC 3783, N III]/N IV is observed to have the value predicted by the models.

\section{DISCUSSION}

The abundance effect discussed here is not the only possible explanation of the BLR density problem. Another likely explanation is that $\mathrm{C} \mathrm{III]}$ emission is coming from a slightly lower density or lower ionization parameter gas that is farther out than the C IV emission. This is supported by many reverberation mapping studies (Gaskell \& Sparke 1986; Koratkar \& Gaskell 1991; Krolik et al. 1991; Reichert et al. 1994; and others).
Integrated emission from clouds with a range of properties also naturally explains integrated line strengths (Baldwin et al. 1995). However, the similarity of C III]/C IV profiles, and hence of the deduced conditions as a function of projected velocity (Snedden \& Gaskell 1999a), argues that C III] and C IV are not coming from very different clouds and that the general abundance/density effect pointed out here is still applicable if the $\mathrm{C} \mathrm{III]}$ and $\mathrm{C}$ IV emission is predominantly coming from different clouds.

We are grateful to Julian Krolik and Hagai Netzer for useful discussions. This work has been supported in part by grant AR-05796.01-94A from the Space Telescope Science Institute, which is operated by AURA, Inc., under NASA contract NAS526555

\section{REFERENCES}

Baldwin, J. A., Ferland, G. J., Korista, K. T., \& Verner, D. A. 1995, ApJ, 455, L119

Ferland, G. J. 1996, HAZY, a Brief Introduction to CLOUDY, Univ. Kentucky Dept. Phys. Astron. Internal Rep.

Ferland, G. J., Baldwin, J. A., Korista, K. T., Hamann, F., Carswell, R. F., Phillips, M. M., Wilkes, B. J., \& Williams, R. E. 1996, ApJ, 461, 683

Ferland, G. J., Korista, K. T., Verner, D. A., Ferguson, J. W., Kingdon, J. B., \& Verner, E. M. 1998, PASP, 110, 761

Gaskell, C. M. 1994, in ASP Conf. Ser. 69, Reverberation Mapping of the Broad-Line Region in Active Galactic Nuclei, ed. P. M. Gondhalekar, K. Horne, \& B. M. Peterson (San Francisco: ASP), 111

Gaskell, C. M., \& Sparke, L. S. 1986, ApJ, 305, 175

Hamann, F., \& Ferland, G. J. 1993, ApJ, 418, 11

Koratkar, A. P., \& Gaskell, C. M. 1991, ApJS, 75, 719

Korista, K. T., Hamann, F. W., Ferguson, J. W., \& Ferland, G. J. 1996, ApJ, 461, 641

Krolik, J. H., Horne, K., Kallman, T. R., Malkan, M. A., Edelson, R. A., \& Kriss, G. A. 1991, ApJ, 371, 541
Kwan, J., \& Krolik, J. H. 1981, ApJ, 250, 478

Mathews, W. G., \& Ferland, G. J. 1987, ApJ, 323, 456

Mushotzky, R. F., \& Ferland, G. J. 1984, ApJ, 278, 558

Osterbrock, D. E. 1970, ApJ, 160, 25

. 1985, in Astrophysics of Active Galaxies and Quasi-Stellar Objects, ed. J. S. Miller (Mill Valley: University Science Books), 111

1989, Astrophysics of Gaseous Nebulae and Active Galactic Nuclei (Mill Valley: University Science Books)

Peterson, B. M., Meyers, K. A., Capriotti, E. R., Foltz, C. B., Wilkes, B. J., \& Miller, H. R. 1985, ApJ, 292, 164

Peterson, B. M., Wanders, I., Horne, K., Collier, S., Alexander, T., Kaspi, S., \& Maoz, D. 1998, PASP, 110, 660

Reichert, G. A., et al. 1994, ApJ, 425, 582

Snedden, S. A. \& Gaskell, C. M. 1999a, in Structure and Kinematics of Quasar Broad-Line Regions, ed. C. M. Gaskell, W. N. Brandt, M. Dietrich, D. Dultzin-Hacyan, \& M. Eracleous (San Francisco: ASP), 25 . 1999 b, in preparation

White, R. J., \& Peterson, B. M. 1994, PASP, 106, 879 